\title{
Wounds at risk of tetanus: unexpected level of underimmunization in a patient cohort
}

\author{
Gaia Bavestrello Piccini ( $\sim$ gaia.bavestrellopic01@universitadipavia.it) \\ UVC Brugmann - Site Victor Horta https://orcid.org/0000-0002-5618-7404 \\ Jean-Christophe Cavenaile \\ UVC Brugmann - Site Victor Horta \\ Maria Antonietta Bressan \\ Fondazione IRCCS Policlinico San Matteo
}

\section{Research article}

Keywords: Tetanus, immunization, prophylaxis, wounds, prevalence, vaccine, immunoglobulin, anamnesis

Posted Date: July 2nd, 2020

DOI: https://doi.org/10.21203/rs.2.20169/v3

License: @) (i) This work is licensed under a Creative Commons Attribution 4.0 International License. Read Full License 


\section{Abstract}

Background: Tetanus is an acute and potentially fatal disease caused by Clostridium tetani, an extremely resilient pathogen. This bacterium can contaminate traumatic wounds which account for approximately $5.4 \%$ of all visits to the Emergency Department. According to several surveillance programs, the incidence of clinical tetanus in Italy is tenfold higher than in other industrialised countries. In 2010, Italy accounted for 57 of the 74 confirmed cases reported within the European Union.

Methods: The study analyses data from 1094 patients who presented to the Emergency Department of the Fondazione IRRCS Policlinico San Matteo between April 2016 and November 2017 with wounds potentially at risk of infection with Clostridium tetani.

Results: Data showed that, in conformity with the literature, the elderly ( $>60$ years old) constitutes a high-risk category, with 219 unprotected individuals out of 238. Also, among patients aged more than 60 years old, there was a statistically significant difference between female and male subjects. From the comparative analysis of the data, it was found that even younger patients are lacking in protective immunity.

Conclusions: When considering other medical systems, both European and non-European ones, and analysing their guidelines for the prevention of tetanus infection, the necessity of adopting a well-defined algorithm becomes evident. This would help avoiding the excessive administration of prophylaxis, as well as putting in place the necessary preventive measures for each patient. This study highlights the need for greater patient and clinician awareness, and for an improvement in record keeping and management of the documentation related to vaccinations. The analysed data also suggest the need for conducting awareness campaigns on the topic of vaccines and vaccine preventable infections. Patients should be made aware of the importance of keeping track of their own immunization status, and of remembering the date of the last administration of the vaccine or, of carrying around their vaccination card.

\section{Background}

Tetanus is an acute and potentially fatal disease caused by infection with the microorganism Clostridium tetani, a spore-forming gram-positive bacterium which is commonly found in the soil of warm and moist areas and may be carried in the intestinal tracts and faeces of humans and animals. These bacteria can get in contact with the internal environment of the human body through a cut, a puncture wound, a burn or a scratch in the skin, which may be superficial ${ }^{[1]}$.

Between 2001 and 2009, a total of 594 tetanus cases were notified in Italy, with an average annual incidence of 1.0/1,000,000 population. The mean annual number of reported deaths was 21. Moreover, the incidence of clinical tetanus in Italy has been shown to be tenfold higher than in other industrialized countries, likely because of higher susceptibility levels ${ }^{[2]}$, since Italy is one of the countries with the highest percentage of elderly population (aged more than 65 ) $^{[3]}$. It has indeed been demonstrated that over $50 \%$ of individuals aged between 45 to 64 and over two thirds of subjects aged more than 65 , have a level of tetanus antibodies lower than $0.01 \mathrm{IU} / \mathrm{ml}{ }^{[2]}$.

In 2010, Italy accounted for 57 of the 74 confirmed cases reported in the EU and it has been continuously reporting the highest number of tetanus cases since 2006 , ranging between 53 and 64 cases per year ${ }^{[4,5]}$.

Although according to a more recent analysis from the European Centre for Disease Prevention and Control there had been a decrease in the reported incidence between 2010 and $2014{ }^{[6]}$, a further increase was observed in 2015, according to a World Health Organization (WHO) survey ${ }^{[7]}$.

Such a trend is probably due to the introduction of the "universal" vaccination campaign for all infants has led to an $86 \%$ incidence reduction between the mid-1950s and the present days ${ }^{[8]}$. Nowadays, in Italy, tetanus affects only subjects who are either unvaccinated or inadequately vaccinated. The purpose of this study was to determinate whether a population at higher risk of not being vaccinated could be outlined, in order to better direct preventive measures for the general population.

\section{Methods}

\section{Study design}

We conducted a retrospective observational study to evaluate the prevalence of vaccination against tetanus in the patient population.

\section{Setting}

The study was conducted at the Fondazione IRCCS (Istituto di Ricovero e Cura a Carattere Scientifico) Policlinico San Matteo. This Hospital is affiliated with the Faculty of Medicine of the University of Pavia (Italy) and is one of the largest teaching hospitals in Italy, hosting every year hundreds of medical students performing clinical rotations.

\section{Participants}


The study included patients presenting to the Emergency Department (ED) between April 2016 and November 2017, with wounds potentially at risk of tetanus infection.

Of the 1094 patients taken into account, 474 (43.33\%) were excluded from the study, due to incompleteness of the digital records, therefore only the medical records of 620 (56.67\%) patients have been taken into account for this study.

\section{Study protocol}

Data on the consecutive ED visits in the aforementioned period, were extracted from electronic patient records using a standard data collection form.

Patients have been subdivided according to their age into 4 categories: 0-18, 19-45, 46-60, 60+. Since the study was carried out between 2016 and 2017 , the subdivision was done considering the age on the $31^{\text {st }}$ December 2016 . Age and nationality have not been considered as exclusion criteria but have been kept into consideration for the subsequent analysis of the results. Exclusion criteria were severely bleeding wounds in need of immediate surgical intervention, and inability to provide a reliable history (i.e. psychiatric disease, dementia or confusion, patient in traumatic shock, unconsciousness).

The type of wound was also recorded, in order to determine the main categories of wounds at higher risk of contamination with $\mathrm{C}$. tetani.

A register of Microsoft Excel was used in order to collect all the data for subsequent epidemiological and statistical analysis.

Comparisons between some of the proportions were performed using a two-sample test for equality of proportions with continuity correction.

\section{Tetanus Quick Stick}

The immune status of patients was determined through the use of the Tetanus Quick Stick ${ }^{\text {TM }}$ (TQS, Zentech, Angleur, Belgium), a point of care testing which allows to conclude whether patients dispose of protecting levels of circulating antibodies against tetanus.

The TQS is an immunochromatographic test which utilizes a combination of tetanus toxoid coated on the solid phase together with a tetanus toxoid dye conjugate for the rapid detection of anti- tetanus antibodies in human serum, plasma or whole blood ${ }^{[9]}$. It is indicated for:

- the determination of the real immune status,

- the identification of unprotected individuals,

- prevention of side reaction due to unnecessary vaccination,

- the follow-up of vaccinated immunodeficient patients

The TQS has been evaluated in several ED worldwide and it has been put into comparison with the gold standard enzyme-linked immunosorbent assay (ELISA). Its sensitivity and specificity have been estimated to be 76 to $88 \%$ and 97 to $100 \%$ respectively ${ }^{[10,11,12]}$, therefore it can be considered as a reliable tool for screening patients that do not need to receive anti-tetanus prophylaxis ${ }^{[13]}$. There are no contraindications to this test ${ }^{[14]}$.

\section{Results}

The dataset contained 620 individuals: 355 males and 265 females; 586 Italians and 34 foreigners.

Out of 620 patients, 114 had not been tested with the TQS for reasons that were not mentioned on the digital records, therefore for some analyses only the sample constituted by the remaining 506 patients (81.61\%) was used.

The statistical analysis of the data has been performed through the Welch two-sample t-test.

Some categories of patients have been found to be at higher risk, for example the elderly ( $>60$ years old), with 219 individuals which tested negative out of the 238 tested. Data revealed a statistically significant difference ( $p$-value $=0.0001)$ between patients aged less than 60 years of age, and those older than 60 years of age (Figure 1 ).

Moreover, among patients aged more than 60 years old, there was a significant difference between female and male patients, with 130 female patients who tested negative and 5 female patients who tested positive, while among the male patients, 89 tested negative and 14 tested positive (Figure 2). This difference was found to be significant ( $p$-value $=0.0095)$ from a statistical point of view, according to the results of the twosample test for equality of proportions with continuity correction.

From the analysis of the data, when taking into consideration the number of patients for every age range who presented with wounds to the ED (Figure 3), it is possible to see that even younger patients are lacking protective immunity (Figure 1, Table 1).

Page $3 / 7$ 
Table 1: Percentage of unprotected patients, according to age

\begin{tabular}{|l|l|l|l|}
\hline \multicolumn{1}{|c|}{ Age } & N. of patients & Patients tested with TQS & \% of negative TQS among tested \\
\hline $60+$ & 271 & 238 & $92 \%$ \\
\hline $46-60$ & 152 & 125 & $80 \%$ \\
\hline $19-45$ & 168 & 128 & $80 \%$ \\
\hline $0-18$ & 29 & 15 & $73 \%$ \\
\hline
\end{tabular}

\section{Discussion}

As reported by other authors, the most important factor associated with a lower immunity rate, was found to be increased age ${ }^{[2,15,16]}$. This is probably due to a combination of the lack of systematic vaccination before 1962, increased life expectancy and lack of administration of the recommended tetanus booster, the decline of tetanus protective antibody levels as age increases, and a deficient immune response to vaccine associated with immunosenescence $[14,15,17,18,19,20]$.

These data confirm the fact that the elderly are the population more at risk since they are less covered by the vaccine, and we can therefore say that the analysed group of patients is representative of the Italian reality as described by the Italian Ministry of Health [16]

For what concerns the category of patients older than 60 years, it is possible to denote a difference among males and females in the immunization rates (Figure 2).

In particular, a slightly higher percentage of male patients of this class (12\%) were protected against tetanus, with respect to female patients (3.7\%). During the next years, in Italy, this finding could undergo a significant change, with a reduction in the difference between males and females aged 60 years or more. This, due to the fact that the Military Service is not obligatory anymore since 2005 [21], but also to the introduction of the obligatory vaccination schedule in $1968^{[22]}$, and to the more recent law ${ }^{[23,24]}$, which reaffirms that for the individuals of age comprised between 0-16 years old, a series of vaccinations has to be rendered mandatory and administered without charge. There will therefore be a greater homogeneity between elderly males and females.

Although the low prevalence of immunization among the elderly can be justified by the aforementioned historical reasons, a more unsettling finding is the one of younger people lacking protective antibody levels.

It is important to focus on the fact that the vaccination against tetanus in Italy is currently mandatory at the age of 3 months, 5 months, 11 months and 6 years in individuals born from 2001 on. A second booster dose is mandatory at 12-18 years for individuals born from 2001 on. Afterwards, Tdap (Tetanus Diphtheria and Pertussis) is recommended every 10 years from age 19, and also for pregnant women in the third trimester (ideally 28 weeks) ${ }^{[25,26]}$. It is therefore astonishing that even the subset of patients aged 0 to 18 years has been partially found to be unprotected against tetanus infection.

The lower rates of protection present even among the younger patients, might be due to factors such as a lack of knowledge about the importance of prevention of this disease through a complete cycle of vaccinations, as well as a lack of awareness on the necessity to receive boosters once completed the primary immunization series. This is probably a consequence of the fact that tetanus is currently one of the most underestimated and less well-known possible complication of a wound.

Another issue which could be important when considering high risk groups populations, is the one of immigration. Even though Pavia is not as cosmopolitan as other cities that have been taken into account by other studies, such as Rome and Brussels, the increase in the number of migrants from other countries in which the healthcare system is not so developed, may be partially responsible for the decrease in vaccination coverage over the next years. In this study, it was not possible to underline major differences for what concerns the difference in immune coverage among patients of different nationalities, due to the small sample of foreign patients (34 units). However, this investigation could be an interesting topic on which to conduct subsequent researches.

In order to increase the prevalence of immunization among patients, prevention should be done, as suggested by the Ministerial Circular concerning the recent Decree Law ${ }^{[27]}$, by promoting vaccinations both for newborns, and more aged patients who somehow did not complete the primary vaccination schedule. A better compliance to vaccine coverage, has also been demonstrated to be associated with fewer hospital admissions in children ${ }^{[28]}$.

Prevention should be done both through notifications under the form of letters, emails, leaflets, but also in a more direct way when the patient presents to the hospital, or to the cabinet of the general practitioner. 
Patients should be made aware of the importance of keeping track of their own immunization status, and of remembering the date of the last administration of the vaccine, and if necessary, the date of the following booster. Moreover, it would be extremely important to more comprehensively educate patients on the topic of vaccines and vaccine preventable infections, stressing on the fact that some of these infections could quickly lead to major complications, and eventually result in the death of the individual.

This study should also render clinicians aware of the fact that not only the elderly could be considered at high risk of not being vaccinated, and all patients with no clear proof of a completed vaccination should undergo a rapid diagnostic test, if available.

\section{Conclusion}

When considering the scattered prevalence of immunization in the population, the necessity of adopting a well-defined algorithm, in order to minimize the error and ensure the best possible care, becomes evident.

Improvements could be done at several levels. First of all, hospitals should adopt protocols as well as effective and cost saving tools. The healthcare personnel should also receive the necessary training in order to put into use these new tools and algorithms in the most effective way. Pharmaceutical companies should be continuously stimulated to discover the best and most effective way to help the diagnostic and therapeutic processes. Ultimately, patients should also become an active party in ameliorating the system, by having a more comprehensive knowledge of their health status.

All this would be important especially for this particular condition, which as this study demonstrated, does not discriminate among individuals of different age, gender, and nationality.

Lastly, as demonstrated from the collected data, as well as the epidemiological data on the trend in the incidence of tetanus infection and in the prevalence of vaccinated individuals in Italy, it is extremely important not to consider this infection as "eradicated", and to never let the guard down on this disease which lurks in the shadow of our mistakes.

\section{Declarations}

- Ethics approval and consent to participate: Informed consent of the patients was deemed not necessary because of the retrospective design of the study, and exemption of ethical approval was granted by the Ethics Committee of the San Matteo Hospital.

- Consent for publication: Not applicable

- Availability of data and material: The datasets used and/or analysed during the current study are available from the corresponding author on reasonable request.

- Competing interests: The authors declare that they have no competing interests

- Funding: Not applicable

- Authors' contributions: GBP: Designed the study, analysed and interpreted data. JCC: Designed and supervised the study, edited multiple manuscript drafts. MAB: collected data. All authors have read and approved the manuscript.

- Acknowledgements: Giovanni Ricevuti, Stefano Perlini, Gabriele Savioli

- Authors' information (optional): Not applicable

\section{List Of Abbreviations}

C. tetani: Clostridium tetani

ED: Emergency Department

TQS: Tetanus Quick Stick

POCT: Point Of Care Testing

ELISA: Enzyme-Linked Immunosorbent Assay

WHO: World Health Organization

IRCCS: Istituto di Ricovero e Cura a Carattere Scientifico

EU: European Union

\section{References}


1. Pellizzari R, Rossetto O, Schiavo G, Montecucco C. Tetanus and botulinum neurotoxins: mechanism of action and therapeutic uses. Philos Trans R Soc Lond B Biol Sci. 1999 Feb 28; 354(1381): 259-268.

2. Filia A, Bella A, von Hunolstein C, et al. Tetanus in Italy 2001-2010: a continuing threat in older adults. Vaccine. 2014;32:639-644.

3. https://data.worldbank.org/indicator/SP.POP.65UP.TO.ZS?end=2018\&most_recent_value_desc=true\&start=2018\&view=map\&year=2018. Accessed 24 Mar 2020

4. European Centre for Disease Prevention and Control. Annual Epidemiological Report 2012. Reporting on 2010 surveillance data and 2011 epidemic intelligence data. Stockholm: ECDC; 2013.

5. http://www.ansa.it/canale_saluteebenessere/notizie/sanita/2017/06/28/vaccini-bambino-senza-copertura-ricoverato-per-tetano_1edb20895bc6-43d1-b522-0b922c03255d.html. Accessed 05 Sep 2017

6. European Centre for Disease Prevention and Control. Annual Epidemiological Report 2016 - Tetanus. Stockholm: ECDC; 2016.

7. WHO/IVB database, data reported to WHO by Member States. http://apps.who.int/ immunization_monitoring/globalsummary/timeseries/tsincidencediphteria.html. Accessed 05 Sep 2017

8. Mandolini D, Ciofi degli Atti M, Pedalino B, Bella A, De Mei B et al. Epidemiology of tetanus in Italy Bollettino Epidemiologico Nazionale; 2002 (3)

9. Information leaflet of the TQS produced by ZenTech. http:// bionicum.com.pl/pliki/pdf/TQS-S-GZ-VZB-001-rev0.pdf. Accessed 20 July 2017

10. Colombet I, Saguez C, Sanson-Le Pors MJ, Coudert B, Chatellier G, Espinoza P: Diagnosis of tetanus immunization status: multicenter assessment of a rapid biological test. Clin Diagn Lab Immunol 2005;12(9):1057-1062

11. Hatamabadi HR, Abdalvand A, Safari S, Kariman H, Dolatabadi AA, et al.Tetanus quick stick as an applicable and cost-effective test in assessment of immunity status. Am J Emerg Med 2011, 29(7):717-720.

12. Paulke-Korinek M, Rendi-Wagner P, Kundi M, Tomann B, Wiedermann U, Kollaritsch H. Pretravel consultation: rapid dipstick test as a decision guidance for the application of tetanus booster vaccinations. Journal of travel medicine 2008;15(6):437-441 Doi: 10.1016/j.it. 2009.05.002 PMC. Web. 29 Sept.2017

13. Golikhatir I, Montazer SH, Bagheri N, et al. Diagnostic Accuracy of Quick Stick for Identifying Traumatic Patients in Need of Tetanus Prophylaxis; a Cross-sectional Study. Emergency 2017;5(1):e66

14. Orsi G, Modini C, Principe M, Di Muzio M, Moriconi A, Amato M, et al. Assessment of tetanus immunity status by tetanus quick stick and anamnesis: a prospective double blind study. Ann Ig. 2015;27(2):467-474.

15. Hainz U, Jeneweih B, Asch E, Pfeiffer KP, Berger P, Grubeck-Loebenstein B. Insufficient protection for healthy elderly adults by tetanus and TBE vaccines. Vaccine 2005; 23(25):3232-3235.

16. Italian Ministry of Health. Decree N. 16 of 11 November 1996. Tetanus prophylaxis measures. (Circolare n.16 del 11/11/1996. Tetano: misure di profilassi).

17. Chen WH, Kozlovsky BF, Effros RB, Grubeck-Loebenstein B, Edelman R, Sztein MB. Vaccination in the elderly: an immunological perspective. Trends in immunology 2009;30(7): 351-359.

18. Michel JP, M. Gusmano, Blank PR, Philp I, Vaccination and healthy ageing: How to make life-course vaccination a successful public health strategy. European Geriatric Medicine 2010;1:155-165.

19. Targonski PV, Jacobson RM, Poland GA. Immunosenescence: role and measurement in influenza vaccine response among the elderly. Vaccine 2007;25:3066-3069

20. Pawelec G, Akbar A, Caruso C, et al. Human immunosenescence: is it infectious? Immunol Rev 2005;205:257-268

21. Legge n. 226 del 23 agosto 2004, in G.U. n. 204 del 31 agosto 2004

22. Legge n. 166 del 27 aprile 1981, in G.U. n. 119 del 2 maggio 1981

23. Decreto legge n. 73 del 7 giugno 2017, in G.U. n. 130 del 7 giugno 2017

24. Legge n. 119 del 31 luglio 2017, in G.U. n. 182 del 5 agosto 2017

25. http://www.salute.gov.it/imgs/C_17_pubblicazioni_2571_allegato.pdf. Accessed 24 Mar 2020

26. https://vaccine-schedule.ecdc.europa.eu/Scheduler/ByCountry?

SelectedCountryld=103\&IncludeChildAgeGroup=true\&IncludeChildAgeGroup=false\&IncludeAdultAgeGroup=true\&IncludeAdultAgeGroup=false . Accessed 24 Mar 2020

27. Italian Ministry of Health. Newsletter 16/08/2017. Urgent instructions regarding prevention through vaccines, infectious diseases and controversies in the administration of drugs (Circolare del 16/08/2017. Disposizioni urgenti in materia di prevenzione vaccinale, di malattie infettive e di controversie relative alla somministrazione di farmaci)

28. Cecil, E., Bottle, A., Ma, R. et al. Impact of preventive primary care on children's unplanned hospital admissions: a population-based birth cohort study of UK children 2000-2013. BMC Med 16, 151 (2018) doi:10.1186/s12916-018-1142-3 
Figures

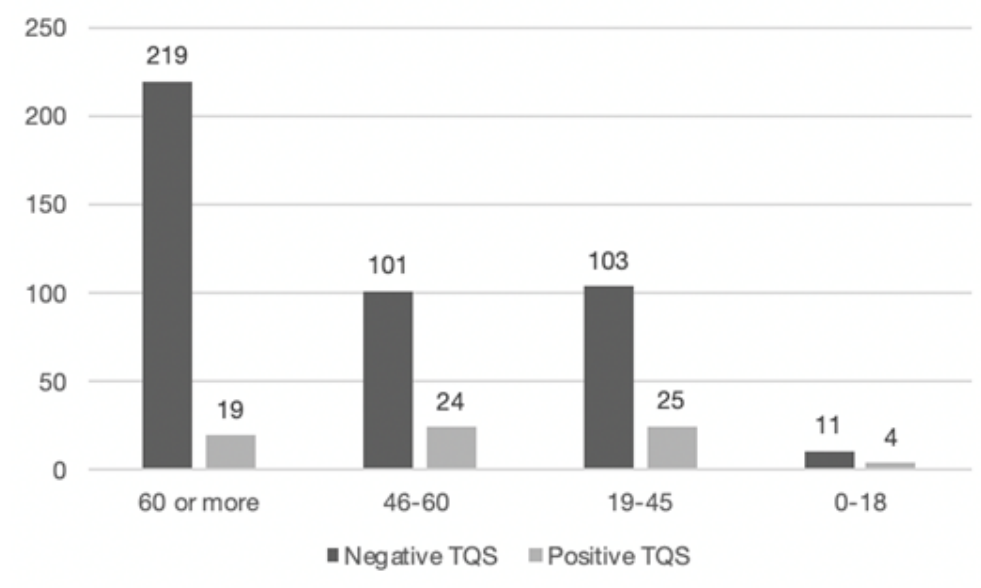

\section{Figure 1}

TQS results with regards to age class of patients

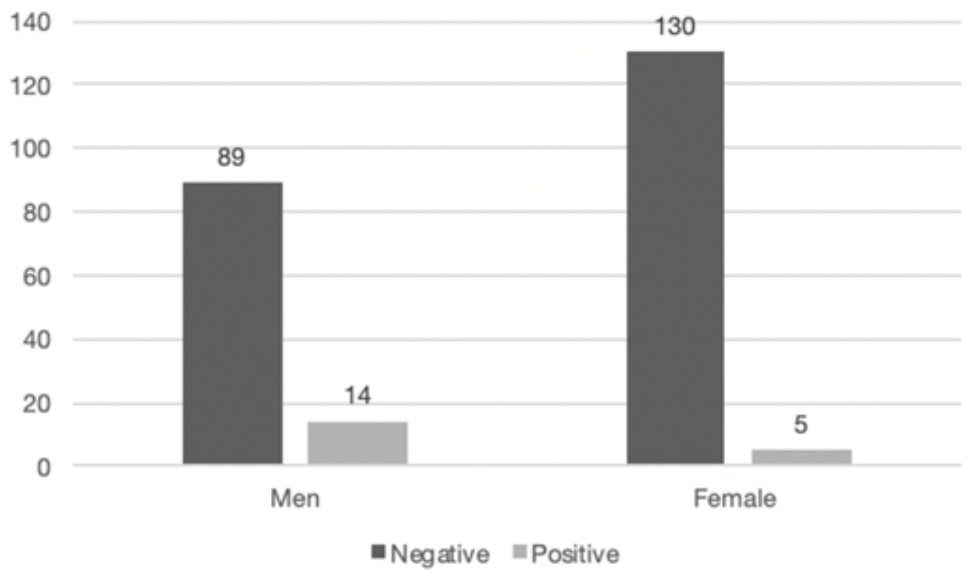

Figure 2

TQS results according to gender in patients aged $>60$

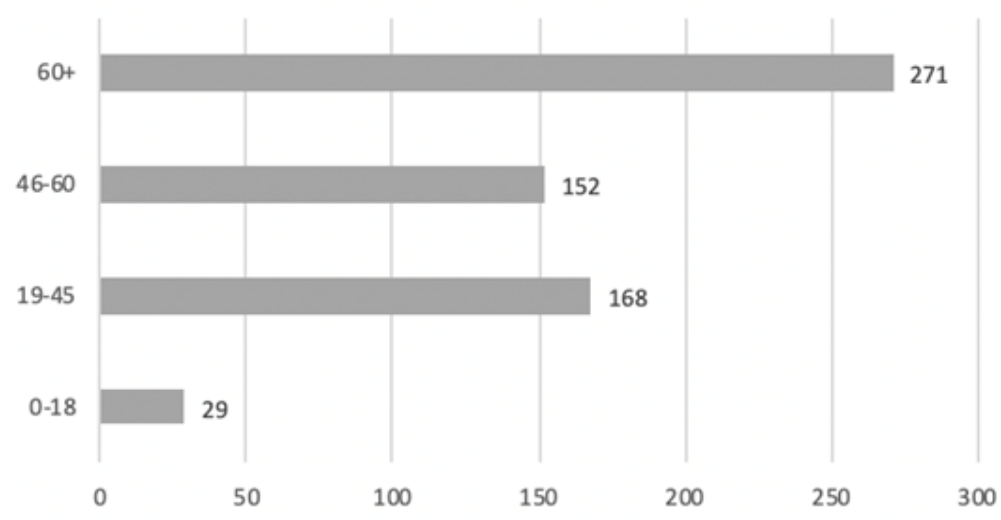

Figure 3

Subdivision of patients accessing the Emergency Department for wounds in age classes 